\title{
Observation of Single Nanocrystal Growth Trajectories in Solution Using Liquid Cell TEM
}

\author{
Haimei Zheng ${ }^{1}$, Hong-Gang Liao ${ }^{1}$, Jungwon Park ${ }^{2}$, Ulrich Dahmen ${ }^{1}$, Paul Alivisatos ${ }^{1,2}$ \\ ${ }^{1}$ Materials Sciences Division, Lawrence Berkeley National Laboratory, Berkeley, CA \\ 94720 \\ ${ }^{2}$ Department of Chemistry, University of California, Berkeley, CA 94720
}

An understanding of the mechanisms of colloidal nanocrystal growth is essential for predictable synthesis of nanocrystals with desired physical properties. In situ observation of the dynamic growth process is expected to advance our understanding of nanocrystal growth mechanisms. By using a self-contained liquid cell operating in a transmission electron microscope (TEM), we have studied in real time the growth of a variety of nanocrystals in solution, including platinum, bismuth, bismuth oxide, bithmuth sulfide, platinum-iron, etc. Using bismuth nanocrystals as a model system for the study, we show the direct imaging of the critical nuclei in solution growth. Size fluctuations of the nuclei have been observed. When an oxide layer is formed at the surface of the bismuth nanocrystals, hollow nanoparticles subsequently formed due to faster outward diffusion of bismuth than inward diffusion of oxygen. Such direct observation of the pathways of ion diffusion will aid in the understanding and control of nanoscale Kirkendall effect. At the end, we will discuss strategies for more quantitative study of solution growth of nanocrytals in a liquid cell.

HZ would like to acknowledge LDRD funding support in LBNL. We thank the facility support from National Center for Electron Microscopy (NCEM) in LBNL. NCEM is funded by the Director, Office of Science, Office of Basic Energy Sciences, Materials Science and Engineering Division of the U.S. Department of Energy under Contract No. DE-AC02-05CH11231. 